\title{
Anticipation in Genetic Diseases: Moving Beyond Nucleotide Repeat Disorders
}

Keywords: Anticipation, Nucleotide repeat, Hereditary spastic paraplegia, HSP, SPG4 doi: $10.1017 /$ cjn.2021.213

Can J Neurol Sci. 2022; 49: 627-628

The discovery of trinucleotide repeat expansions as a pathogenic molecular mechanism in the early 1990s was a major shift from the genetic inheritance norms of those days, based on the transmission of stable DNA sequences. The possibility that a gene configuration may differ from one generation to the next, and that this circumstance may correlate with increasing molecular instability, has also provided the basis to elucidate the phenomenon of anticipation. ${ }^{1}$

Prior to this, anticipation, as the description of a pattern of inheritance where a hereditary illness strikes earlier and with increased severity and penetrance in succeeding generations, had been observed for more than a century and had no explanation under established principles of heredity. ${ }^{2}$ The term, in fact, was coined by the British ophthalmologist Edward Nettleship in the early 20th Century as a seemingly eugenic rule of nature aimed to repair diseased lineages by concentrating and exacerbating the illness in certain members of the family while leaving others unharmed. ${ }^{2}$ Over the following decades, anticipation was observed in Huntington disease, myotonic dystrophy and Leber hereditary optic neuropathy. ${ }^{3}$ These purely clinical perceptions led, however, to disagreement amongst researchers as some of them rejected the mere existence of true anticipation due to the lack of a biological rationale.

Under the light of the aforementioned discovery of nucleotide repeat expansion mechanism, anticipation as part of a phenotype was initially linked to unstable genotypes, namely, fragile $\mathrm{X}$ syndrome (FMR1 gene - CGG repeat expansion), X-linked bulbospinal muscle atrophy (androgen receptor gene - CAG repeat expansion) and myotonic dystrophy (DMPK gene - CTG repeat expansion). ${ }^{4}$ Later, the connection was also established for other repeat expansion diseases: spinocerebellar ataxias types 1 , 2, 3, 6, 7, 12 and 17 (CAG repeat expansion), dentatorubropallidoluysian atrophy (ANT1 gene - CAG repeat expansion), frontotemporal dementia and/or amyotrophic lateral sclerosis (C9orf72 gene - GGGGCC repeat expansion), as well as diseases not linked to unstable molecular defects, such as dyskeratosis congenita with TERC and TERT mutations, cancer and autoimmune diseases susceptibility genes, and, questionably, among families with autosomal dominant hereditary spastic paraplegia (HSP, loci designated as SPG) types 3, 4, 6, 9, 10, 12,29 and $31 .^{5-7}$ With regard to the HSPs, although all of these observations remain speculative and derived from a limited number of families, SPG3 and SPG4 stand out as the ones in which this phenomenon has been more consistently observed. ${ }^{8}$

In this issue of the Journal, Hashemi et al. ${ }^{9}$ showed further evidence of anticipation among multiple generations in five Iranian families with SPG4, incorporating their data into findings derived from other families with this form of HSP previously described in the literature. The authors studied a total of 14 unrelated autosomal dominant HSP probands initially using whole exome sequencing, complemented by in silico and segregation analyses, and multiplex ligation-dependent probe amplification. A total of five probands with pathogenic SPAST gene mutations were identified. The variants found were then screened in available family members and cosegregated with the disease status. In these large pedigrees, that included 39 affected individuals spanning up to four generations, the authors were able to collect in-depth detailed demographic and clinical data through personal interviews and/or previous medical records of all affected and unaffected members. Using strict criteria for age of onset across multiple generations, the authors observed unequivocal anticipation with progressive decline in age of onset in later generations of all pedigrees, ranging from a decrease from 47 to 23 years within two generations in one family to from $\sim 60$ to 2.5 years in four generations in another family. These observations were significant and consistent throughout all five pedigrees and across all generations, but absent in the remaining nine families with mutations in other genes or among genetically undiagnosed families. Also, although there was considerable intra- and inter-familial heterogeneity for a few aspects of SPG4 phenotype, age of onset and progression of disability were consistently less variable among affected siblings.

These findings are intriguing given that, among the families described by the authors and those reviewed from the literature in their study, anticipation was observed in pedigrees diagnosed with static mutations in the SPAST gene in which molecular analysis confirmed that neither the effect of the mutations nor their molecular configuration changed from one generation to another. ${ }^{10}$ As such, Hashemi et al. ${ }^{9}$ present a thorough discussion on the potential hypothesis behind this finding, including molecular mechanisms, environmental factors, methodological issues (i.e., ascertainment bias for age of onset of symptoms), most of which not applicable to the families they describe. The discussion also addresses why anticipation might have been missed in many families with SPG4 reported in the literature.

If confirmed, the implications of these findings are potentially significant. First, they increase the growing body of literature with respect to the phenotype (possibly allowing phenotype/ genotype correlation) of HSPs and SPG4 in particular. Second, these observations may be the groundwork for better understanding the molecular dysfunction leading to SPG4, for unveiling the mechanism behind the phenomenon of anticipation in 
diseases linked to seemingly stable/static mutations, and even for the discovery of novel treatment strategies. Third, they provide vital elements for discussion regarding genetic counselling, prognosis and clinical course of this disease in symptomatic and presymptomatic mutation carriers. Finally, once understood, such a mechanism may allow us to hypothesize how it may underlie observations such as intrafamilial heterogeneity and apparent sporadic occurrence of autosomal dominant disorders.

\section{Disclosures}

The author has no conflicts of interest to declare.

\section{Renato Puppi Munhoz}

Edmond J. Safra Program in Parkinson's Disease and Morton and Gloria Shulman Movement Disorders Clinic, Toronto Western Hospital, UHN, Division of Neurology, University of Toronto, Toronto, Ontario, Canada Krembil Brain Institute, Toronto, Ontario, Canada

Correspondence to: Renato P. Munhoz, Edmond J. Safra Program in Parkinson's Disease and Morton and Gloria Shulman Movement Disorders Clinic, Toronto Western Hospital, 399 Bathurst Street, Toronto, ON M5T 2S8, Canada. Email: renato.munhoz@uhn.ca

\section{REFERENCES}

1. Mahadevan M, Tsilfidis C, Sabourin L, et al. Myotonic dystrophy mutation: an unstable CTG repeat in the 3' untranslated region of the gene. Science. 1992;255:1253-55.

2. Khristich AN, Mirkin SM. On the wrong DNA track: molecular mechanisms of repeat-mediated genome instability. J Biol Chem. 2020;295:4134-70.

3. Friedman JE. Anticipation in hereditary disease: the history of a biomedical concept. Hum Genet. 2011;130:705-14.

4. La Spada AR, Paulson HL, Fischbeck KH. Trinucleotide repeat expansion in neurological disease. Ann Neurol. 1994;36: 814-22.

5. Depienne C, Mandel JL. 30 years of repeat expansion disorders: what have we learned and what are the remaining challenges? Am J Hum Genet. 2021;108:764-85.

6. Munhoz RP, Kawarai T, Teive HA, et al. Clinical and genetic study of a Brazilian family with spastic paraplegia (SPG6 locus). Mov Disord. 2006;21:279-81.

7. Kamada M, Kawarai T, Miyamoto R, et al. Spastic paraplegia type 31: a novel REEP1 splice site donor variant and expansion of the phenotype variability. Parkinsonism Relat Disord. 2018;46:79-83.

8. Bürger J, Fonknechten N, Hoeltzenbein M, et al. Hereditary spastic paraplegia caused by mutations in the SPG4 gene. Eur J Hum Genet. 2000;8:771-76.

9. Hashemi SS, Hajati R, Davarzani A, et al. Anticipation can be more common in hereditary spastic paraplegia with SPAST mutations than it appears. Can J Neurol Sci. 2021;49:651-61.

10. Iwai K, Yamamoto M, Yoshihara T, Sobue G. Anticipation in familial amyotrophic lateral sclerosis with SOD1-G93S mutation. J Neurol Neurosurg Psychiatry. 2002;72:819-20. 